\title{
AN OPTIMAL ORDER YIELDING DISCREPANCY PRINCIPLE FOR SIMPLIFIED REGULARIZATION OF ILL-POSED PROBLEMS IN HILBERT SCALES
}

\author{
SANTHOSH GEORGE and M. THAMBAN NAIR
}

Received 19 March 2002

\begin{abstract}
Recently, Tautenhahn and Hämarik (1999) have considered a monotone rule as a parameter choice strategy for choosing the regularization parameter while considering approximate solution of an ill-posed operator equation $T x=y$, where $T$ is a bounded linear operator between Hilbert spaces. Motivated by this, we propose a new discrepancy principle for the simplified regularization, in the setting of Hilbert scales, when $T$ is a positive and selfadjoint operator. When the data $y$ is known only approximately, our method provides optimal order under certain natural assumptions on the ill-posedness of the equation and smoothness of the solution. The result, in fact, improves an earlier work of the authors (1997).
\end{abstract}

2000 Mathematics Subject Classification: 65J20, 65R30, 65J15, 47A52, 47J06.

1. Introduction. Tikhonov regularization (cf. [2]) is one of the most widely used procedures to obtain stable approximate solution to an ill-posed operator equation

$$
T x=y,
$$

where $T: X \rightarrow Y$ is a bounded linear operator between Hilbert spaces $X$ and $Y$. Suppose that the data $y$ is not exactly known, but only an approximation of it, namely $\tilde{y}$, is available. Then, the regularized solution $\tilde{x}_{\alpha}$, by Tikhonov regularization, is obtained by minimizing the map

$$
x \longmapsto\|T x-\tilde{y}\|^{2}+\alpha\|x\|^{2}
$$

for $\alpha>0$. For $y \in R(T)+R(T)^{\perp}$, if $\hat{x}$ is the generalized solution of (1.1), that is, $\hat{x}=T^{\dagger} y$, where $T^{\dagger}$ is the Moore-Penrose generalized inverse of $T$, then estimates for the error $\left\|\hat{x}-\tilde{x}_{\alpha}\right\|$ are obtained by choosing the regularization parameter $\alpha$ appropriately. It is known that (see, e.g., [2]) if $\hat{x} \in R\left(\left(T^{*} T\right)^{v}\right)$ for some $v>0$ and if $\|y-\tilde{y}\| \leq \delta$ for some noise level $\delta>0$, then the optimal order for the above error is $O\left(\delta^{\mu}\right)$, where $\mu=\min \{2 v /(2 v+1), 2 / 3\}$.

In order to improve the error estimates available in Tikhonov regularization, Natterer [9] carried out error analysis in the framework of Hilbert scales. Subsequently, many authors extended, modified, and generalized Natterer's work 
to obtain error bounds under various contexts (see, e.g., Natterer [9], Hegland [3], Schröter and Tautenhahn [12], Mair [6], Nair et al. [8], and Nair [7]).

If $T$ is a positive and selfadjoint operator on a Hilbert space, then the simplified regularization introduced by Lavrentiev is better suited than Tikhonov regularization in terms of speed of convergence and condition number in the case of finite-dimensional approximations (cf. Schock [11]).

In [1], simplified regularization in the framework of Hilbert scales was studied for the first time and obtained error estimates under a priori and a posteriori parameter choice strategies. The a posteriori choice of the parameter in that paper has a drawback that it can yield the optimal rate only under certain restricted smoothness assumption on the solution.

In this paper, we propose a new discrepancy principle, for choosing the regularization parameter $\alpha$, for simplified regularization in the setting of Hilbert scales, which eliminates the drawback of the method in [1] yielding the optimal order for a range of values of smoothness. The discrepancy principle of this paper is motivated by a recent procedure adopted by Tautenhahn and Hämarik [13].

2. Preliminaries. Let $H$ be a Hilbert space and let $A: H \rightarrow H$ be a bounded, positive and selfadjoint operator on $H$. Recall that $A$ is said to be a positive operator if $\langle A x, x\rangle \geq 0$ for every $x \in H$. For $y \in R(A)$, the range of $A$, consider the operator equation

$$
A x=y
$$

Let $\hat{x}$ be the minimal norm solution of (2.1). It is well known that if $R(A)$ is not closed in $H$, then the problem of solving (2.1) for $\hat{x}$ is ill-posed in the sense that small perturbation in the data $y$ can cause large deviations in the solution.

A prototype of (2.1) is an integral equation of the first kind,

$$
\int_{0}^{1} k(s, t) x(t) d t=y(s), \quad 0 \leq s \leq 1,
$$

where $k(\cdot, \cdot)$ is a nondegenerate kernel which is square integrable, that is,

$$
\int_{0}^{1} \int_{0}^{1}|k(s, t)|^{2} d t d s<\infty
$$

satisfying $k(s, t)=k(t, s)$ for all $s, t$ in $[0,1]$, and such that the eigenvalues of the corresponding integral operator $A: L^{2}[0,1] \rightarrow L^{2}[0,1]$,

$$
(A x)(s)=\int_{0}^{1} k(s, t) x(t) d t, \quad 0 \leq s \leq 1,
$$


are all nonnegative. For example, consider the kernel $k(\cdot, \cdot)$ defined by

$$
k(s, t)= \begin{cases}(1-s) t, & \text { if } 0 \leq s \leq t \leq 1 \\ (1-t) s, & \text { if } 0 \leq t \leq s \leq 1\end{cases}
$$

Clearly, $k(s, t)=k(t, s)$, so that $A: L^{2}[0,1] \rightarrow L^{2}[0,1]$, defined as in (2.4), is a selfadjoint operator. Moreover, the eigenvalues of this operator are $1 / n^{2} \pi^{2}$ for $n=1,2, \ldots$ (see Limaye [5, page 329]).

For considering the regularization of (2.1) in the setting of Hilbert scales, we consider a Hilbert scale $\left\{H_{t}\right\}_{t \in \mathbb{R}}$ generated by a strictly positive operator $L: D(L) \rightarrow H$ with its domain $D(L)$ dense in $H$ satisfying

$$
\|L x\| \geq\|x\|, \quad x \in D(L) .
$$

Recall (cf. [4]) that the space $H_{t}$ is the completion of $D:=\bigcap_{k=0}^{\infty} D\left(L^{k}\right)$ with respect to the norm $\|x\|_{t}$, induced by the inner product

$$
\langle u, v\rangle_{t}=\left\langle L^{t} u, L^{t} v\right\rangle, \quad u, v \in D
$$

Moreover, if $\beta \leq \gamma$, then the embedding $H_{\gamma} \hookrightarrow H_{\beta}$ is continuous, and therefore the norm $\|\cdot\|_{\beta}$ is also defined in $H_{\gamma}$ and there is a constant $c_{0,1}$ such that

$$
\|x\|_{\beta} \leq c_{0,1}\|x\|_{\gamma}, \quad x \in H_{\gamma} .
$$

We assume that the ill-posed nature of the operator $A$ is related to the Hilbert scale $\left\{H_{t}\right\}_{t \in \mathbb{R}}$ according to the relation

$$
c_{1}\|x\|_{-a} \leq\|A x\| \leq c_{2}\|x\|_{-a}, \quad x \in H,
$$

for some positive reals $a, c_{1}$, and $c_{2}$.

For the example of an integral operator given in the previous paragraph, one may take $L$ to be defined by

$$
L x:=\sum_{j=1}^{\infty} j^{2}\left\langle x, u_{j}\right\rangle u_{j},
$$

where $u_{j}(t):=\sqrt{2} \sin (j \pi t), j \in \mathbb{N}$, and the domain of $L$ is

$$
D(L):=\left\{x \in L^{2}[0,1]: \sum_{j=1}^{\infty} j^{4}\left|\left\langle x, u_{j}\right\rangle\right|^{2}<\infty\right\} .
$$

In this case, it can be seen that

$$
H_{t}=\left\{x \in L^{2}[0,1]: \sum_{j=1}^{\infty} j^{4 t}\left|\left\langle x, u_{j}\right\rangle\right|^{2}<\infty\right\}
$$


and the constants $a, c_{1}$, and $c_{2}$ in (2.9) are given by $a=1$ and $c_{1}=c_{2}=1 / \pi^{2}$ (see Schröter and Tautenhahn [12, Section 4]).

As in [1], we consider the regularized solution of (1.1) as the solution of the well-posed equation

$$
\left(A+\alpha L^{s}\right) x_{\alpha}=y, \quad \alpha>0,
$$

where $s$ is a fixed nonnegative real number.

Suppose that the data $y \neq 0$ is known only approximately, say $\tilde{y} \neq 0$ with $\|y-\tilde{y}\| \leq \delta$ for a known error level $\delta>0$. Then, in place of (2.13), we consider

$$
\left(A+\alpha L^{s}\right) \tilde{x}_{\alpha}=\tilde{y} .
$$

It can be seen that the solution $\tilde{x}_{\alpha}$ of the above equation is the unique minimizer of the function

$$
x \longmapsto\langle A x, x\rangle-2\langle\tilde{y}, x\rangle+\alpha\left\langle L^{s} x, x\right\rangle, \quad x \in D(L) .
$$

We also observe that taking

$$
A_{S}:=L^{-s / 2} A L^{-s / 2} \text {, }
$$

(2.13) and (2.14) take the forms

$$
L^{s / 2}\left(A_{s}+\alpha I\right) L^{s / 2} x_{\alpha}=y, \quad L^{s / 2}\left(A_{s}+\alpha I\right) L^{s / 2} \tilde{x}_{\alpha}=\tilde{y}
$$

respectively. Note that the operator $A_{s}$ defined above is positive and selfadjoint bounded operator on $H$.

One of the crucial results for proving the results in [1] as well as the results in this paper is the following result, where functions $f$ and $g$ are defined by

$$
f(t)=\min \left\{c_{1}^{t}, c_{2}^{t}\right\}, \quad g(t)=\max \left\{c_{1}^{t}, c_{2}^{t}\right\}, \quad t \in \mathbb{R},|t| \leq 1,
$$

respectively.

Proposition 2.1 (see [1, Proposition 3.1]). For $s \geq 0$ and $|v| \leq 1$,

$$
f\left(\frac{v}{2}\right)\|x\|_{-v(s+a) / 2} \leq\left\|A_{s}^{v / 2} x\right\| \leq g\left(\frac{v}{2}\right)\|x\|_{-v(s+a) / 2}, \quad x \in H .
$$

Using the above proposition, the following result has been proved in [1].

THEOREM 2.2 (see [1, Theorem 3.2]). Suppose that $\hat{x} \in H_{t}, 0<t \leq s+a$, and $\alpha>0$. Then

$$
\left\|\hat{x}-\tilde{x_{\alpha}}\right\| \leq \phi(s, t) \alpha^{t /(s+a)}\|x\|_{t}+\psi(s) \alpha^{-a /(s+a)} \delta
$$


where

$$
\phi(s, t)=\frac{g((s-2 t) /(2 s+2 a))}{f(s /(2 s+2 a))}, \quad \psi(s)=\frac{g(-s /(2 s+2 a))}{f(s /(2 s+2 a))} .
$$

In particular, if $\alpha=c_{0} \delta^{(s+a) /(t+a)}$ for some constant $c_{0}>0$, then

$$
\left\|\hat{x}-\tilde{x_{\alpha}}\right\| \leq \eta(s, t) \delta^{t /(t+a)}
$$

where

$$
\eta(s, t)=\max \left\{\phi(s, t)\|\hat{x}\|_{t} c_{0}^{t /(t+a)}, \psi(s) c_{0}^{-a /(s+a)}\right\} .
$$

Let $R_{\alpha}=\left(A_{s}+\alpha I\right)^{-1}$. We will make use of the relation

$$
\left\|R_{\alpha} A_{s}^{\top}\right\| \leq \alpha^{\tau-1}, \quad \alpha>0,0<\tau \leq 1
$$

which follows from the spectral properties of the selfadjoint operator $A_{s}, s>0$.

In [1], the authors considered parameter choice strategies, a priori and a posteriori, which yield the optimal rate $O\left(\delta^{t /(t+a)}\right)$ if $\hat{x} \in H_{t}$ for certain specific values of $t$. The a posteriori parameter choice strategy in [1] is to choose $\alpha$ such that

$$
\alpha^{p+1}\left\|\left(A_{s}+\alpha I\right)^{-p-1} L^{-s / 2} x\right\|=k \delta
$$

where $k>1$ and $\tilde{y} \in X$ satisfy $0<k \delta \leq\|\tilde{y}\|_{-s / 2}$. Under the above procedure, the optimal order $O\left(\delta^{t /(t+a)}\right)$ is obtained for $t=s+p(s+a)$.

In the present paper, we propose a new discrepancy for choosing the regularization parameter $\alpha$ which yields the optimal rate

$$
\left\|\hat{x}-\tilde{x_{\alpha}}\right\|=O\left(\delta^{t /(t+a)}\right)
$$

3. The discrepancy principle. Let $s$ and $a$ be fixed positive real numbers. For $\alpha>0$ and nonzero $x \in H$, let

$$
\Phi(\alpha, x):=\frac{\alpha\left\|R_{\alpha}^{3 / 2} A_{s}^{-s /(2 s+2 a)} L^{-s / 2} x\right\|^{2}}{\left\|R_{\alpha}^{2} A_{s}^{-s /(2 s+2 a)} L^{-s / 2} x\right\|} .
$$

Note that, by assumption (2.9), $\left\|R_{\alpha}^{2} A_{s}^{-s /(2 s+2 a)} L^{-s / 2} x\right\|$ is nonzero for every nonzero $x \in H$ so that the function $\Phi(\alpha, x)$ is well defined for every $\alpha>0$ and for every nonzero $x \in H$.

We assume that the available data $\tilde{y}$ is nonzero and

$$
\|y-\tilde{y}\| \leq \delta
$$


for some known error level $\delta>0$. Our idea is to prove the existence of a unique $\alpha$ such that

$$
\Phi(\alpha, \tilde{y})=c \delta
$$

for some known $c>0$.

In due course we will make use of the relation

$$
f\left(\frac{-s}{2 s+2 a}\right)\|x\| \leq\left\|A_{s}^{-s /(2 s+2 a)} L^{-s / 2} x\right\| \leq g\left(\frac{-s}{2 s+2 a}\right)\|x\|
$$

which can be easily derived from Proposition 2.1.

First we prove the monotonicity of the function $\Phi(\alpha, x)$ defined in (3.1).

THEOREM 3.1. For each nonzero $x \in H$, the function $\alpha \mapsto \Phi(\alpha, x)$ for $\alpha>0$, defined in (3.1), is increasing and it is continuously differentiable with $\Phi^{\prime}(\alpha, x) \geq$ 0 . In addition

$$
\lim _{\alpha \rightarrow 0} \Phi(\alpha, x)=0, \quad \lim _{\alpha \rightarrow \infty} \Phi(\alpha, x)=\left\|A_{s}^{-s /(2 s+2 a)} L^{-s / 2} x\right\|
$$

PROoF. Using (3.1), one can write

$$
\begin{aligned}
\frac{d}{d \alpha} \Phi( & \alpha, x) \\
= & \frac{(d / d \alpha)\left(\Phi^{2}(\alpha, x)\right)}{2 \Phi(\alpha, x)} \\
= & \frac{2 \alpha\left\|R_{\alpha}^{2} A_{s}^{-s /(2 s+2 a)} L^{-s / 2} x\right\|^{2}\left\|R_{\alpha}^{3 / 2} A_{s}^{-s /(2 s+2 a)} L^{-s / 2} x\right\|^{2}}{2 \alpha\left\|R_{\alpha}^{3 / 2} A_{s}^{-s /(2 s+2 a)} L^{-s / 2} x\right\|^{2}} \\
& \times \frac{(d / d \alpha)\left[\alpha\left\|R_{\alpha}^{3 / 2} A_{s}^{-s /(2 s+2 a)} L^{-s / 2} x\right\|^{2}\right]}{\left\|R_{\alpha}^{2} A_{s}^{-s /(2 s+2 a)} L^{-s / 2} x\right\|^{3}} \\
& -\frac{\alpha^{2}\left\|R_{\alpha}^{3 / 2} A_{s}^{-s /(2 s+2 a)} L^{-s / 2} x\right\|^{4}(d / d \alpha)\left[\left\|R_{\alpha}^{2} A_{s}^{-s /(2 s+2 a)} L^{-s / 2} x\right\|^{2}\right]}{2 \alpha\left\|R_{\alpha}^{3 / 2} A_{s}^{-s /(2 s+2 a)} L^{-s / 2} x\right\|^{2}\left\|R_{\alpha}^{2} A_{s}^{-s /(2 s+2 a)} L^{-s / 2} x\right\|^{3}} .
\end{aligned}
$$

Thus,

$$
\begin{aligned}
\frac{d}{d \alpha} \Phi(\alpha, x) & \\
= & \frac{\left\|R_{\alpha}^{2} A_{s}^{-s /(2 s+2 a)} L^{-s / 2} x\right\|^{2}(d / d \alpha)\left[\alpha\left\|R_{\alpha}^{3 / 2} A_{s}^{-s /(2 s+2 a)} L^{-s / 2} x\right\|^{2}\right]}{\left\|R_{\alpha}^{2} A_{s}^{-s /(2 s+2 a)} L^{-s / 2} x\right\|^{3}} \\
& -\frac{\alpha\left\|R_{\alpha}^{3 / 2} A_{s}^{-s /(2 s+2 a)} L^{-s / 2} x\right\|^{2}(d / d \alpha)\left[\left\|R_{\alpha}^{2} A_{s}^{-s /(2 s+2 a)} L^{-s / 2} x\right\|^{2}\right]}{2\left\|R_{\alpha}^{2} A_{s}^{-s /(2 s+2 a)} L^{-s / 2} x\right\|^{3}} .
\end{aligned}
$$


Let $\left\{E_{\lambda}: 0 \leq \lambda \leq a\right\}$ be the spectral family of $A_{s}$, where $a=\left\|A_{s}\right\|$. Then

$$
\begin{aligned}
& \frac{d}{d \alpha}\left(\alpha\left\|R_{\alpha}^{3 / 2} A_{s}^{-s /(2 s+2 a)} L^{-s / 2} x\right\|^{2}\right) \\
& \quad=\frac{d}{d \alpha} \int_{0}^{a} \frac{\alpha}{\lambda^{s /(s+a)}(\lambda+\alpha)^{3}} d\left\langle E_{\lambda} L^{-s / 2} x, L^{-s / 2} x\right\rangle \\
& \quad=\int_{0}^{a}\left[\frac{1}{\lambda^{s /(s+a)}(\lambda+\alpha)^{3}}-\frac{3 \alpha}{\lambda^{s /(s+a)}(\lambda+\alpha)^{4}}\right] d\left\langle E_{\lambda} L^{-s / 2} x, L^{-s / 2} x\right\rangle \\
& =\left\|R_{\alpha}^{3 / 2} A_{s}^{-s /(2 s+2 a)} L^{-s / 2} x\right\|^{2}-3 \alpha\left\|R_{\alpha}^{2} A_{s}^{-s /(2 s+2 a)} L^{-s / 2} x\right\|^{2} .
\end{aligned}
$$

Similarly

$$
\frac{d}{d \alpha}\left(\left\|R_{\alpha}^{2} A_{s}^{-s /(2 s+2 a)} L^{-s / 2} x\right\|\right)=-4\left\|R_{\alpha}^{5 / 2} A_{s}^{-s /(2 s+2 a)} L^{-s / 2} x\right\|^{2} .
$$

Therefore, from (3.7), using (3.8) and (3.9), we get

$$
\begin{aligned}
\frac{d}{d \alpha} \Phi( & \alpha, x) \\
= & \left\|R_{\alpha}^{2} A_{s}^{-s /(2 s+2 a)} L^{-s / 2} x\right\|^{2} \\
& \times \frac{\left[\left\|R_{\alpha}^{3 / 2} A_{s}^{-s /(2 s+2 a)} L^{-s / 2} x\right\|^{2}-3 \alpha\left\|R_{\alpha}^{2} A_{s}^{-s /(2 s+2 a)} L^{-s / 2} x\right\|^{2}\right]}{\left\|R_{\alpha}^{2} A_{s}^{-s /(2 s+2 a)} L^{-s / 2} x\right\|^{3}} \\
& +\frac{2 \alpha\left\|R_{\alpha}^{3 / 2} A_{s}^{-s /(2 s+2 a)} L^{-s / 2} x\right\|^{2}\left\|R_{\alpha}^{5 / 2} A_{s}^{-s /(2 s+2 a)} L^{-s / 2} x\right\|^{2}}{\left\|R_{\alpha}^{2} A_{s}^{-s /(2 s+2 a)} L^{-s / 2} x\right\|^{3}} .
\end{aligned}
$$

The above equation can be rewritten as

$$
\frac{d}{d \alpha} \Phi(\alpha, x)=\Psi_{1}(\alpha, x)+\Psi_{2}(\alpha, x)
$$

where

$$
\begin{aligned}
& \Psi_{1}(\alpha, x) \\
& =\left\|R_{\alpha}^{2} A_{s}^{-s /(2 s+2 a)} L^{-s / 2} x\right\|^{2} \\
& \quad \times \frac{\left[\left\|R_{\alpha}^{3 / 2} A_{s}^{-s /(2 s+2 a)} L^{-s / 2} x\right\|^{2}-\alpha\left\|R_{\alpha}^{2} A_{s}^{-s /(2 s+2 a)} L^{-s / 2} x\right\|^{2}\right]}{\left\|R_{\alpha}^{2} A_{s}^{-s /(2 s+2 a)} L^{-s / 2} x\right\|^{3}}, \\
& \Psi_{2}(\alpha, x) \\
& =\left(2 \alpha \left[\left\|R_{\alpha}^{3 / 2} A_{s}^{-s /(2 s+2 a)} L^{-s / 2} x\right\|^{2}\right.\right. \\
& \left.\left.\quad \times\left\|R_{\alpha}^{5 / 2} A_{s}^{-s /(2 s+2 a)} L^{-s / 2} x\right\|^{2}-\left\|R_{\alpha}^{2} A_{s}^{-s /(2 s+2 a)} L^{-s / 2} x\right\|^{4}\right]\right) \\
& \quad \times \frac{1}{\left\|R_{\alpha}^{2} A_{s}^{-s /(2 s+2 a)} L^{-s / 2} x\right\|^{3}} .
\end{aligned}
$$


Since

$$
\begin{aligned}
& \left\|R_{\alpha}^{3 / 2} A_{s}^{-s /(2 s+2 a)} L^{-s / 2} x\right\|^{2} \\
& \quad=\left\langle\left(A_{s}+\alpha I\right)^{-3} A_{s}^{-s /(2 s+2 a)} L^{-s / 2} x, A_{s}^{-s /(2 s+2 a)} L^{-s / 2} x\right\rangle, \\
& \left\|R_{\alpha}^{2} A_{s}^{-s /(2 s+2 a)} L^{-s / 2} x\right\|^{2} \\
& \quad=\left\langle\left(A_{s}+\alpha I\right)^{-3} A_{s}^{-s /(2 s+2 a)} L^{-s / 2} x,\left(A_{s}+\alpha I\right)^{-1} A_{s}^{-s /(2 s+2 a)} L^{-s / 2} x\right\rangle,
\end{aligned}
$$

we have

$$
\begin{gathered}
\left\|R_{\alpha}^{3 / 2} A_{s}^{-s /(2 s+2 a)} L^{-s / 2} x\right\|^{2}-\alpha\left\|R_{\alpha}^{2} A_{s}^{-s /(2 s+2 a)} L^{-s / 2} x\right\|^{2} \\
=\left\|A_{s}^{a /(2 s+2 a)} R_{\alpha}^{2} L^{-s / 2} x\right\|^{2} .
\end{gathered}
$$

Also,

$$
\begin{aligned}
\| R_{\alpha}^{2} & A_{s}^{-s /(2 s+2 a)} L^{-s / 2} x \|^{4} \\
& =\left[\left\langle R_{\alpha}^{2} A_{s}^{-s /(2 s+2 a)} L^{-s / 2} x, R_{\alpha}^{2} A_{s}^{-s /(2 s+2 a)} L^{-s / 2} x\right\rangle\right]^{2} \\
& =\left[\left\langle R_{\alpha}^{3 / 2} A_{s}^{-s /(2 s+2 a)} L^{-s / 2} x, R_{\alpha}^{5 / 2} A_{s}^{-s /(2 s+2 a)} L^{-s / 2} x\right\rangle\right]^{2} \\
& \leq\left\|R_{\alpha}^{3 / 2} A_{s}^{-s /(2 s+2 a)} L^{-s / 2} x\right\|^{2}\left\|R_{\alpha}^{5 / 2} A_{s}^{-s /(2 s+2 a)} L^{-s / 2} x\right\|^{2} .
\end{aligned}
$$

Hence

$$
\Psi_{1}(\alpha, x) \geq 0, \quad \Psi_{2}(\alpha, x) \geq 0
$$

so that

$$
\frac{d}{d \alpha}(\Phi(\alpha, x))=\Psi_{1}(\alpha, x)+\Psi_{2}(\alpha, x) \geq 0
$$

To prove the last part of the theorem we observe that

$$
\begin{aligned}
& \alpha^{2}\left\|R_{\alpha}^{2} A_{s}^{-s /(2 s+2 a)} L^{-s / 2} x\right\|-\Phi(\alpha, x) \\
& =\frac{\alpha^{2}\left\|R_{\alpha}^{2} A_{s}^{-s /(2 s+2 a)} L^{-s / 2} x\right\|^{2}-\alpha\left\|R_{\alpha}^{3 / 2} A_{s}^{-s /(2 s+2 a)} L^{-s / 2} x\right\|^{2}}{\left\|R_{\alpha}^{2} A_{s}^{-s /(2 s+2 a)} L^{-s / 2} x\right\|} .
\end{aligned}
$$

Since

$$
\begin{aligned}
& \alpha^{2}\left\|R_{\alpha}^{2} A_{s}^{-s /(2 s+2 a)} L^{-s / 2} x\right\|^{2} \\
& =\alpha\left\langle R_{\alpha}^{3} A_{s}^{-s /(2 s+2 a)} L^{-s / 2} x, \alpha R_{\alpha} A_{s}^{-s /(2 s+2 a)} L^{-s / 2} x\right\rangle, \\
& \alpha\left\|R_{\alpha}^{3 / 2} A_{s}^{-s /(2 s+2 a)} L^{-s / 2} x\right\|^{2} \\
& \quad=\alpha\left\langle R_{\alpha}^{3} A_{s}^{-s /(2 s+2 a)} L^{-s / 2} x, A_{s}^{-s /(2 s+2 a)} L^{-s / 2} x\right\rangle,
\end{aligned}
$$


and since $\alpha R_{\alpha}-I=A_{s} R_{\alpha}=R_{\alpha} A_{s}$, we have

$$
\begin{aligned}
\alpha^{2}\left\|R_{\alpha}^{2} A_{s}^{-s /(2 s+2 a)} L^{-s / 2} x\right\|-\Phi(\alpha, x) \\
=\frac{-\alpha\left\langle R_{\alpha}^{3} A_{s}^{-s /(2 s+2 a)} L^{-s / 2} x, A_{s} R_{\alpha} A_{s}^{-s /(2 s+2 a)} L^{-s / 2} x\right\rangle}{\left\|R_{\alpha}^{2} A_{s}^{-s /(2 s+2 a)} L^{-s / 2} x\right\|} \\
=\frac{-\alpha\left\|A_{s}^{a /(2 s+2 a)} R_{\alpha}^{2} L^{-s / 2} x\right\|^{2}}{\left\|R_{\alpha}^{2} A_{s}^{-s /(2 s+2 a)} L^{-s / 2} x\right\|} \leq 0 .
\end{aligned}
$$

Hence

$$
\Phi(\alpha, x) \geq \alpha^{2}\left\|R_{\alpha}^{2} A_{s}^{-s /(2 s+2 a)} L^{-s / 2} x\right\| \geq \alpha^{2} \frac{\left\|A_{s}^{-s /(2 s+2 a)} L^{-s / 2} x\right\|}{\left(\left\|A_{s}\right\|+\alpha\right)^{2}} .
$$

Also, we have

$$
\begin{aligned}
\Phi(\alpha, x) & =\frac{\alpha\left\langle R_{\alpha} A_{s}^{-s /(2 s+2 a)} L^{-s / 2} x, R_{\alpha}^{2} A_{s}^{-s /(2 s+2 a)} L^{-s / 2} x\right\rangle}{\left\|R_{\alpha}^{2} A_{s}^{-s /(2 s+2 a)} L^{-s / 2} x\right\|} \\
& \leq \alpha\left\|R_{\alpha} A_{s}^{-s /(2 s+2 a)} L^{-s / 2} x\right\| .
\end{aligned}
$$

Hence

$$
\begin{aligned}
& \left(\frac{\alpha}{\left\|A_{s}\right\|+\alpha}\right)^{2}\left\|A_{s}^{-s /(2 s+2 a)} L^{-s / 2} x\right\| \\
& \quad \leq \Phi(\alpha, x) \leq \alpha\left\|R_{\alpha} A_{s}^{-s /(2 s+2 a)} L^{-s / 2} x\right\| .
\end{aligned}
$$

From this, it follows that

$$
\lim _{\alpha \rightarrow 0} \Phi(\alpha, x)=0, \quad \lim _{\alpha \rightarrow \infty} \Phi(\alpha, x)=\left\|A_{s}^{-s /(2 s+2 a)} L^{-s / 2} x\right\| .
$$

This completes the proof.

For the next theorem, in addition to (3.2), we assume that

$$
\left\|A_{s}^{-s /(2 s+2 a)} L^{-s / 2} \tilde{y}\right\| \geq c \delta
$$

for some $c>0$. This assumption will be satisfied if, for example,

$$
\delta \leq \frac{\tilde{f}(s)}{c+\tilde{f}(s)}\|y\|, \quad \tilde{f}(s):=f\left(\frac{-s}{2 s+2 a}\right)
$$

since, by (3.2), we have $\|\tilde{y}\| \geq\|y\|-\delta$, and by (3.4),

$$
\left\|A_{s}^{-s /(2 s+2 a)} L^{-s / 2} \tilde{y}\right\| \geq f\left(\frac{-s}{2 s+2 a}\right)\|\tilde{y}\|,
$$

where $f$ is as in (2.18).

Now, the following theorem is a consequence of Theorem 3.1. 
THEOREM 3.2. Assume that (3.2) and (3.25) are satisfied. Then there exists a unique $\alpha:=\alpha(\delta)$ satisfying

$$
\Phi(\alpha, \tilde{y})=c \delta
$$

4. Error estimates. In order to obtain Hölder-type error bounds, that is, error

bounds of the form

$$
\left\|\tilde{x_{\alpha}}-\hat{x}\right\|=O\left(\delta^{\tau}\right)
$$

for some $\tau$, we assume that the solution $\hat{x}$ of (2.1) satisfies the source condition (as in $[1,10])$ :

$$
\hat{x} \in M_{\rho, t}:=\left\{x \in H_{t}:\|x\|_{t} \leq \rho\right\}
$$

for some $t>0$.

LEMMA 4.1. Suppose that $\hat{x}$ belongs to $M_{\rho, t}$ for some $t \leq s$, and $\alpha:=\alpha(\delta)>0$ is the unique solution of (3.28), where $c>g(-s /(2 s+2 a))$. Then

$$
\alpha \geq c_{0} \delta^{(s+a) /(t+a)}, \quad c_{0}=\frac{c-g(-s /(2 s+2 a))}{g((s-2 t) /(2 s+2 a)) \rho} .
$$

Proof. Note that by (3.22), Proposition 2.1, and (2.24), we have

$$
\begin{aligned}
\Phi(\alpha, \tilde{y}) \leq & \alpha\left\|R_{\alpha} A_{s}^{-s /(2 s+2 a)} L^{-s / 2} \tilde{y}\right\| \\
\leq & \alpha\left\|R_{\alpha} A_{s}^{-s /(2 s+2 a)} L^{-s / 2}(\tilde{y}-y)\right\|+\alpha\left\|R_{\alpha} A_{s}^{-s /(2 s+2 a)} A_{s} L^{s / 2} \hat{x}\right\| \\
\leq & \alpha\left\|R_{\alpha} A_{s}^{-s /(2 s+2 a)} L^{-s / 2}(\tilde{y}-y)\right\|+\alpha\left\|R_{\alpha} A_{s}^{(s+2 a) /(2 s+2 a)} L^{s / 2} \hat{x}\right\| \\
\leq & \alpha\left\|R_{\alpha} A_{s}^{-s /(2 s+2 a)} L^{-s / 2}(\tilde{y}-y)\right\| \\
& +\alpha\left\|R_{\alpha} A_{s}^{(t+a) /(s+a)} A_{s}^{(s-2 t) /(2 s+2 a)} L^{s / 2} \hat{x}\right\| \\
\leq & \left\|\alpha R_{\alpha}\right\|\left\|A_{s}^{-s /(2 s+2 a)} L^{-s / 2}(\tilde{y}-y)\right\| \\
& +\left\|\alpha R_{\alpha} A_{s}^{(t+a) /(s+a)}\right\|\left\|A_{s}^{(s-2 t) /(2 s+2 a)} L^{s / 2} \hat{x}\right\| \\
\leq & g\left(\frac{-s}{2 s+2 a}\right) \delta+g\left(\frac{s-2 t}{2 s+2 a}\right) \rho \alpha^{(t+a) /(s+a)} .
\end{aligned}
$$

Thus

$$
\left[c-g\left(\frac{-s}{2 s+2 a}\right)\right] \delta \leq g\left(\frac{s-2 t}{2 s+2 a}\right) \rho \alpha^{(t+a) /(s+a)},
$$

which implies

$$
\alpha \geq c_{0} \delta^{(s+a) /(t+a)}, \quad c_{0}=\frac{c-g(-s /(2 s+2 a))}{g((s-2 t) /(2 s+2 a)) \rho} .
$$

This completes the proof. 
THEOREM 4.2. Under the assumptions in Lemma 4.1,

$$
\left\|\hat{x}-x_{\alpha}\right\|=O\left(\delta^{\kappa}\right), \quad \kappa:=\frac{t}{t+a} .
$$

Proof. Since $x_{\alpha}$ is the solution of (2.13), we have

$$
\begin{aligned}
\hat{x}-x_{\alpha} & =\hat{x}-\left(A+\alpha L^{s}\right)^{-1} y \\
& =\alpha L^{-s / 2}\left(A_{s}+\alpha I\right)^{-1} L^{s / 2} \hat{x}=\alpha L^{-s / 2} R_{\alpha} L^{s / 2} \hat{x} .
\end{aligned}
$$

Therefore, by (3.4), we have

$$
f\left(\frac{s}{2 s+2 a}\right)\left\|\hat{x}-x_{\alpha}\right\| \leq\left\|\alpha A_{s}^{s /(2 s+2 a)} R_{\alpha} L^{s / 2} \hat{x}\right\| .
$$

To obtain an estimate for $\left\|\alpha A_{s}^{s /(2 s+2 a)} R_{\alpha} L^{s / 2} \hat{x}\right\|$, first we will make use of the following moment inequality

$$
\left\|B^{u} x\right\| \leq\left\|B^{v} x\right\|^{u / v}\|x\|^{1-u / v}, \quad 0 \leq u \leq v,
$$

where $B$ is a positive selfadjoint operator. Precisely, we use (4.10) with

$$
\begin{gathered}
u=\frac{t}{a}, \quad v=1+\frac{t}{a}, \quad B=\alpha R_{\alpha} A_{s}^{a /(s+a)}, \\
x=\alpha^{1-t / a} R_{\alpha}^{1-t / a} A_{s}^{(s-2 t) /(2 s+2 a)} L^{s / 2} \hat{x} .
\end{gathered}
$$

Then since

$$
\begin{aligned}
\|x\| & \leq\left\|A_{s}^{(s-2 t) /(2 s+2 a)} L^{s / 2} \hat{x}\right\| \\
& \leq g\left(\frac{s-2 t}{2 s+2 a}\right)\left\|L^{s / 2} \hat{x}\right\|_{t-s / 2} \leq g\left(\frac{s-2 t}{2 s+2 a}\right) \rho,
\end{aligned}
$$

we have

$$
\begin{aligned}
& \left\|\alpha A_{s}^{s /(2 s+2 a)} R_{\alpha} L^{s / 2} \hat{x}\right\| \\
& \quad=\left\|B^{t / a} x\right\| \leq\left\|B^{1+t / a} x\right\|^{t /(t+a)}\|x\|^{a /(t+a)} \\
& \quad \leq\left\|\alpha^{2} R_{\alpha}^{2} A_{s}^{(2 a+s) /(2 s+2 a)} L^{s / 2} \hat{x}\right\|^{t /(t+a)}\|x\|^{a /(t+a)} \\
& \quad \leq\left\|\alpha^{2} R_{\alpha}^{2} A_{s}^{-s /(2 s+2 a)} L^{-s / 2} y\right\|^{t /(t+a)}\|x\|^{a /(t+a)} \\
& \quad \leq g\left(\frac{s-2 t}{2 s+2 a}\right)^{a /(t+a)} \rho^{a /(t+a)}\left\|\alpha^{2} R_{\alpha}^{2} A_{s}^{-s /(2 s+2 a)} L^{-s / 2} y\right\|^{t /(t+a)} .
\end{aligned}
$$

Further, by (2.24) and (3.20),

$$
\begin{aligned}
\left\|\alpha^{2} R_{\alpha}^{2} A_{s}^{-s /(2 s+2 a)} L^{-s / 2} y\right\| \leq & \left\|\alpha^{2} R_{\alpha}^{2} A_{s}^{-s /(2 s+2 a)} L^{-s / 2}(y-\tilde{y})\right\| \\
& +\left\|\alpha^{2} R_{\alpha}^{2} A_{s}^{-s /(2 s+2 a)} L^{-s / 2} \tilde{y}\right\| \\
\leq & \delta+\Phi(\alpha, \tilde{y}) .
\end{aligned}
$$


Therefore, if $\alpha:=\alpha(\delta)$ is the unique solution of (3.28), then we have

$$
\left\|\alpha^{2} R_{\alpha}^{2} A_{s}^{-s /(2 s+2 a)} L^{-s / 2} y\right\| \leq(1+c) \delta .
$$

Now the result follows from (4.9), (4.13), (4.14), and (4.15).

THEOREM 4.3. Under the assumptions in Lemma 4.1,

$$
\left\|\hat{x}-\tilde{x}_{\alpha}\right\|=O\left(\delta^{\kappa}\right), \quad \kappa:=\frac{t}{t+a} .
$$

Proof. Let $x_{\alpha}$ and $\tilde{x}_{\alpha}$ be the solutions of (2.13) and (2.14), respectively. Then by triangle inequality, (2.24), and Proposition 2.1,

$$
\begin{aligned}
\left\|\hat{x}-\tilde{x}_{\alpha}\right\| & \leq\left\|\hat{x}-x_{\alpha}\right\|+\left\|x_{\alpha}-\tilde{x}_{\alpha}\right\| \\
& =\left\|\hat{x}-x_{\alpha}\right\|+\left\|L^{-s / 2} R_{\alpha} L^{-s / 2}(y-\tilde{y})\right\| \\
& \leq\left\|\hat{x}-x_{\alpha}\right\|+\frac{1}{f(s /(2 s+2 a))}\left\|A_{s}^{s /(2 s+2 a)} R_{\alpha} L^{-s / 2}(y-\tilde{y})\right\| \\
& \leq\left\|\hat{x}-x_{\alpha}\right\|+\frac{1}{f(s /(2 s+2 a))}\left\|A_{s}^{s /(s+a)} R_{\alpha} A_{s}^{-s /(2 s+2 a)} L^{-s / 2}(y-\tilde{y})\right\| \\
& \leq\left\|\hat{x}-x_{\alpha}\right\|+\frac{1}{f(s /(2 s+2 a))}\left\|A_{s}^{s /(s+a)} R_{\alpha}\right\|\left\|A_{s}^{-s /(2 s+2 a)} L^{-s / 2}(y-\tilde{y})\right\| \\
& \leq\left\|\hat{x}-x_{\alpha}\right\|+\frac{g(-s /(2 s+2 a))}{f(s /(2 s+2 a))} \delta \alpha^{-a /(s+a)} .
\end{aligned}
$$

The proof now follows from Lemma 4.1 and Theorem 4.2.

REMARK 4.4. We observe that unlike the discrepancy principle in [1], the discrepancy principle (3.3) gives the optimal order $O\left(\delta^{t /(t+a)}\right)$ for all $0<t \leq s$.

ACKNOWLEDGments. The first author acknowledges the assistance from the Institute of Mathematical Sciences Chennai, India, for providing the opportunity to work in the institute as an Associate Member. The author also thanks Dr. M. Krishna for his help.

\section{REFERENCES}

[1] S. George and M. T. Nair, Error bounds and parameter choice strategies for simplified regularization in Hilbert scales, Integral Equations Operator Theory 29 (1997), no. 2, 231-242.

[2] C. W. Groetsch, The Theory of Tikhonov Regularization for Fredholm Equations of the First Kind, Research Notes in Mathematics, vol. 105, Pitman, Massachusetts, 1984.

[3] M. Hegland, An optimal order regularization method which does not use additional smoothness assumptions, SIAM J. Numer. Anal. 29 (1992), no. 5, 1446-1461.

[4] S. G. Krein and J. I. Petunin, Scales of Banach spaces, Russian Math. Surveys 21 (1966), no. 2, 85-160. 
[5] B. V. Limaye, Functional Analysis, 2nd ed., New Age International Publishers, New Delhi, 1996.

[6] B. A. Mair, Tikhonov regularization for finitely and infinitely smoothing operators, SIAM J. Math. Anal. 25 (1994), no. 1, 135-147.

[7] M. T. Nair, On Morozov's method for Tikhonov regularization as an optimal order yielding algorithm, Z. Anal. Anwendungen 18 (1999), no. 1, 37-46.

[8] M. T. Nair, M. Hegland, and R. S. Anderssen, The trade-off between regularity and stability in Tikhonov regularization, Math. Comp. 66 (1997), no. 217, 193-206.

[9] F. Natterer, Error bounds for Tikhonov regularization in Hilbert scales, Applicable Anal. 18 (1984), no. 1-2, 29-37.

[10] A. Neubauer, An a posteriori parameter choice for Tikhonov regularization in Hilbert scales leading to optimal convergence rates, SIAM J. Numer. Anal. 25 (1988), no. 6, 1313-1326.

[11] E. Schock, Ritz-regularization versus least-square-regularization. Solution methods for integral equations of the first kind, Z. Anal. Anwendungen 4 (1985), no. 3, 277-284.

[12] T. Schröter and U. Tautenhahn, Error estimates for Tikhonov regularization in Hilbert scales, Numer. Funct. Anal. Optim. 15 (1994), no. 1-2, 155-168.

[13] U. Tautenhahn and U. Hämarik, The use of monotonicity for choosing the regularization parameter in ill-posed problems, Inverse Problems 15 (1999), no. 6, 1487-1505.

Santhosh George: Department of Mathematics, Government College, Sanquelim, Goa 403505, India

E-mail address: santhoshsq1729@yahoo.co.in

M. Thamban Nair: Department of Mathematics, Indian Institute of Technology, Madras, Chennai 600 036, India

E-mail address: mtnair@i itm.ac.in 


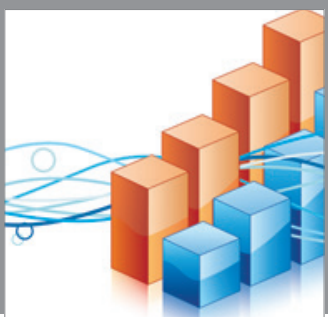

Advances in

Operations Research

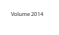

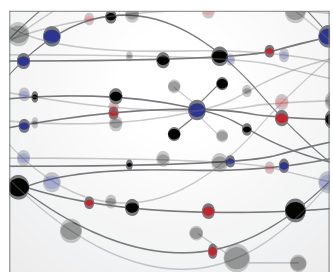

\section{The Scientific} World Journal
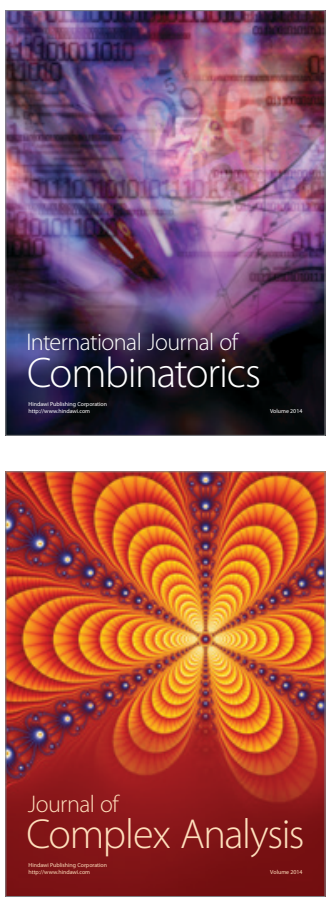

International Journal of

Mathematics and

Mathematical

Sciences
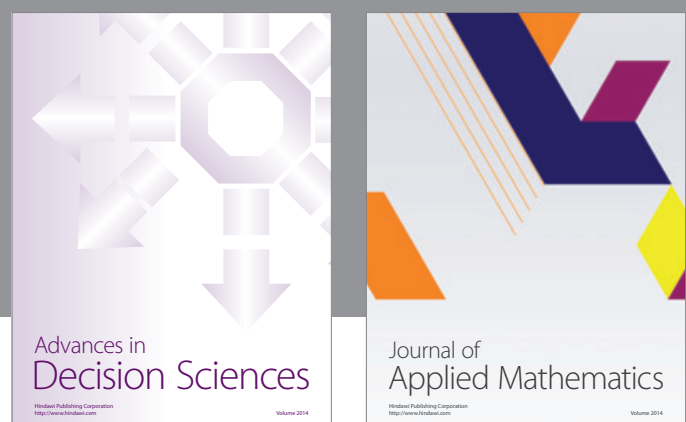

Journal of

Applied Mathematics
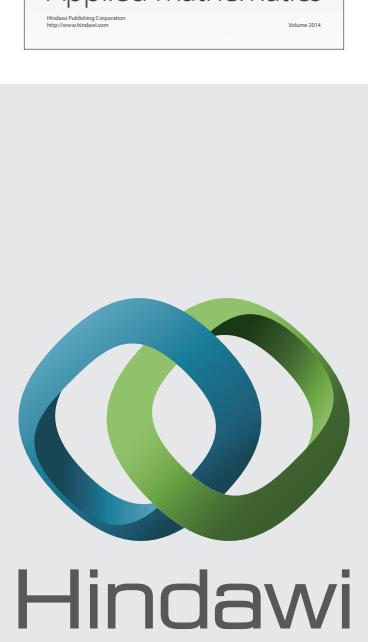

Submit your manuscripts at http://www.hindawi.com
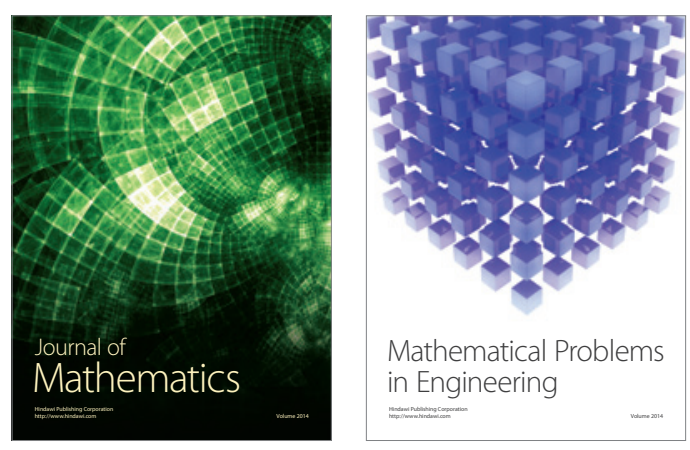

Mathematical Problems in Engineering
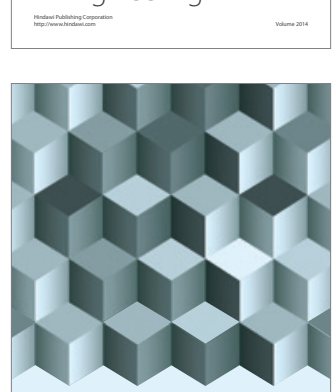

Journal of

Function Spaces
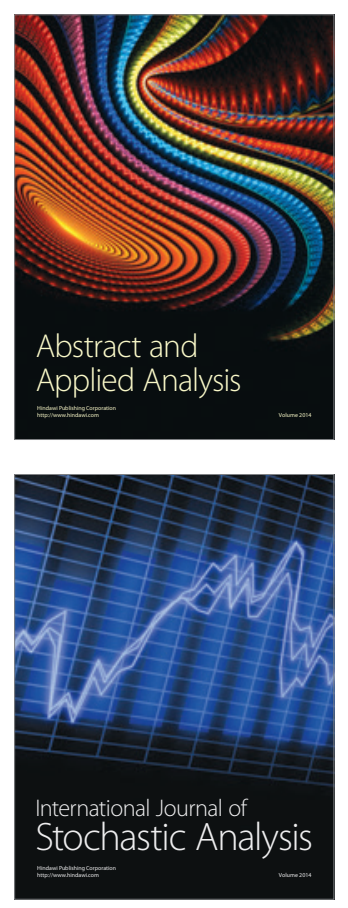

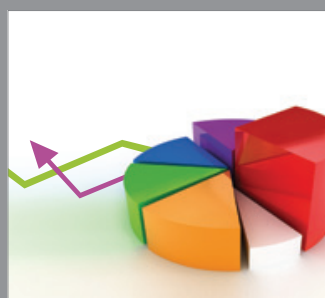

ournal of

Probability and Statistics

Promensencen
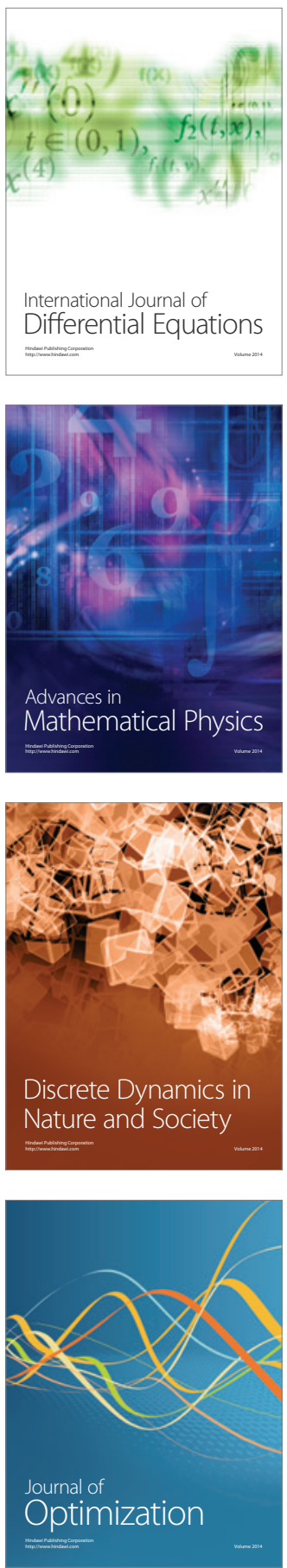